age-related cataracts. These reports have suggested that high and low BMIs can affect the onset or progression of age-related visual impairment. However, few prospective studies have examined this relationship in a general Asian population. Therefore, in this study, we investigated whether BMI was associated with increased risk of age-related cataracts by performing a 5 -year prospective populationbased study among a middle-aged Japanese population.

Methods This 5-year population-based study included 35365 men and 40825 women (aged 45-74), who were recruited onto the Japan Public Health Center-based Prospective Study (JPHC Study) and had not reported cataracts in baseline survey. The self-reported diagnosis of age-related cataracts was used in the analysis of this study.

Results At follow-up, 1004 men (2.84\%) and 1807 women (4.43\%) reported new diagnoses of age-related cataracts. The multivariate ORs for those in the lowest and the highest BMI category, compared with a BMI category of $21.0-22.9$ as a reference point (OR, 1.00), were 1.29 (95\% CI 0.93 to 1.79$)$ and 1.15 (95\% CI 0.96 to 1.39$)$ in men, and 1.23 (95\% CI 0.97 to 1.55$)$ and 1.19 (95\% CI 1.04 to 1.36$)$ in women.

Conclusion High and low BMIs have been suggested previously as the risk of age-related cataracts for Caucasian population in developed countries and the population living in developing countries respectively. However, the present large-cohort study showed that a $\mathrm{U}$-shaped association between BMI and incidence of cataracts in Japanese men and women.

\section{P2-339 ROLE OF NEUROMUSCULAR FUNCTION IN PREDICTING THE OCCURRENCE OF DISABILITY: THE ROAD STUDY}

\section{doi:10.1136/jech.2011.142976k.71}

${ }^{1} \mathrm{~N}$ Yoshimura, ${ }^{*}{ }^{1} \mathrm{~S}$ Muraki, ${ }^{1} \mathrm{H}$ Oka, ${ }^{2} \mathrm{H}$ Kawaguchi, ${ }^{2} \mathrm{~K}$ Nakamura, ${ }^{1} \mathrm{~T}$ Akune. ${ }^{1} 22 n d$ Century Medical and Research Center, The University of Tokyo, Tokyo, Japan; ${ }^{2}$ Department of Orthopaedic Surgery, The University of Tokyo, Tokyo, Japan

Introduction To assess the ability of neuromuscular functions to predict the occurrence of disability in Japanese general population. Methods The large-scale population-based cohort study entitled research on osteoarthritis/osteoporosis against disability (ROAD) has established three cohorts, from an urban, a mountainous, and a coastal area. The first follow-up survey was performed after 3 years and was attended by 2479 of 3040 baseline participants (81.5\%). From the entire ROAD data, we utilised the data of the elderly participants ( $\geq 65$ years) from the mountainous and coastal cohorts. Disability was defined by a relevant certification committee comprising clinical experts and physicians from each municipality. To evaluate the predictive ability of the indices of neuromuscular function, logistic regression analysis was conducted using occurrence of disability as an objective factor and the grip strength and walking speed for $6 \mathrm{~m}$ as explanatory factors after adjustment for age, gender, and body mass index.

Results Of the 914 participants who completed both baseline and first follow-up surveys, 88 individuals $(9.6 \%)$ were defined as new cases of disability over 3 years. Logistic regression analysis indicated that the higher the grip strength, the lower is the risk of disability (+1 kg; OR, 0.90; 95\% CI 0.86 to 0.95), whereas the lesser the walking speed, the greater is the risk $(+1 \mathrm{~s}$; OR, 1.15; CI 1.07 to 1.23).

Conclusions The cumulative incidence of disability among the elderly was $9.6 \%$ over 3 years. The indices of neuromuscular function may be potential predictive factors of the occurrence of disability over 3 years.

\section{P2-340 HARMONY IN FAMILY RELATIONS: EXPLORATORY ANALYSIS IN SEARCH OF A HARMONY SCALE}

doi:10.1136/jech.2011.142976k.72

Y-Y Yu, W Tam, T H Lam. ${ }^{*}$ The University of Hong Kong, Hong Kong, Hong Kong

Objective Relationship harmony is characteristic of Chinese values and has been increasingly identified as a factor that contributes to social stability. However, no existing survey instrument is available that assesses the concept of family harmony in a Chinese context. We aimed to develop an opinion scale that captures self-evaluated state of family harmony among Hong Kong Chinese.

Methods During June 2008-October 2008, we surveyed 587 Cantonese-speaking Hong Kong residents (aged 15 and above) randomly selected from a list of residential addresses. We collected demographic data and asked the participants how much they agree with a pool of 52 descriptive items on family relations, family functions, respect, loyalty, love and support, communications and conflict resolution. Most items were positively worded indicative good family relations except five reverse-coded items. Responses ranged from strongly agree (1) to strongly disagree (5). We used exploratory factor analysis with maximum likelihood extraction method.

Results Score distributions for all 52 items were similar, the majority of the participants "agreed" to most items. Mean score of the 52 items was $2.2(\mathrm{SE}=0.4)$. Exploratory factor analysis identified nine dimensions of the harmony construct with Eigen values above unity, which explained $59 \%$ of total variance, and two dimensions with Eigen values of at least two, which explained $48 \%$ of total variance.

Conclusion Small variation was found for each of the 52 opinion items on family relations harmony. After item reduction, more indepth interviews are needed to examine whether these opinion items truly reflect the state of family harmony.

\section{P2-341 THE PREVALENCE AND INFLUENCES ON AETIOLOGY OF ANGINA IN RURAL AND URBAN POPULATIONS IN A DEVELOPING COUNTRY: THE PERU MIGRANT STUDY}

doi:10.1136/jech.2011.142976k.73

${ }^{1} \mathrm{M}$ Justin Zaman, ${ }^{*}{ }^{2} \mathrm{C} \mathrm{L}$ de Mola, ${ }^{3,2} \mathrm{R}$ H Gilman, ${ }^{4} \mathrm{~L}$ Smeeth, ${ }^{2,4} \mathrm{~J} \mathrm{~J}$ Miranda. ${ }^{1}$ University College London, London, UK; ${ }^{2}$ CRONICAS Center of Excellence in Chronic Diseases, Universidad Peruana Cayetano Heredia, Lima, Peru; ${ }^{3} J o h n s$ Hopkins Bloomberg School of Public Health, Baltimore, USA; ${ }^{4}$ London School of Hygiene and Tropical Medicine, London, UK

Background Rural-to-urban migration in low- and middle-income countries causes an increase in individual cardiovascular risk. There are few data on prevalence of early stage coronary diseases such as angina in developing countries, while the understanding of the aetiology of angina is complicated by the difficulty in measuring it across differing populations.

Methods The PERU MIGRANT study was designed to investigate differences between rural-to-urban migrant and non-migrant groups in specific cardiovascular disease risk factors cross-sectionally. The Rose angina questionnaire was used to record chest pain, which was classified definite, possible and non-exertional. Mental health was measured using the General Health Questionnaire (GHO-12). Mantel-Haenszel ORs (adjusted for age, sex, cardiovascular disease risk factors and mental health) were used to assess the risk of chest pain in the migrant and urban groups compared to the rural group, and further to assess the relationship (age and sex-adjusted) between risk factors, mental health and chest pain. 
Results Compared to the urban group, rural dwellers had a greatly increased likelihood of possible/definite angina (multi-adjusted OR 2.82 (1.68 to 4.73)). Urban and migrant groups had higher levels of risk factors (eg, smoking - 20.1\% urban, 5.5\% rural). No diabetes was seen in the rural dwellers who complained of possible/definite angina. Rural dwellers had a higher prevalence of mood disorder and the presence of a mood disorder was associated with possible/definite angina in all three groups, but not consistently with nonexertional chest pain.

Conclusion Rural groups had a higher prevalence of angina as measured by Rose questionnaire than migrants and urban dwellers, and a higher prevalence of mood disorder. The presence of a mood disorder was associated with angina. The Rose angina questionnaire may not be of relevance to rural populations in developing countries with a low pre-test probability of coronary disease and poor mental health.

\section{P2-342 GEOGRAPHICAL PATTERN AND EPIDEMIOLOGY OF STOMACH CANCER MORTALITY IN IRAN}

\section{doi:10.1136/jech.2011.142976k.74}

\author{
${ }^{1,2} \mathrm{~K}$ Zendehdel, ${ }^{*}{ }^{1} \mathrm{M}$ Marzban, ${ }^{1} \mathrm{~A}$ Nahvijou, ${ }^{1} \mathrm{~N}$ Jafari. ${ }^{1}$ Cancer Research Center, \\ Cancer Institute of Iran, Tehran, Iran; ${ }^{2}$ Department of Medical Epidemiology and \\ Biostatistcs, Karolinska Institutet, Stockholm, Sweden; ${ }^{3}$ Health Network Development \\ Center, Ministry of Health and Medical Education, Tehran, Iran
}

Background Stomach cancer is the second commonest cause of mortality from cancer worldwide; in Iran it is the commonest. Geographical variation in the incidence of stomach cancer is reported. Data describing the geographical distribution of disease in Iran are lacking. The aim of this study was to examine geographical variation in stomach cancer mortality in Iran.

Methods We used the Iranian National Causes of Death Registry and estimated age-standardised mortality rates (ASMR) of stomach cancer in 29 provinces, using the age distribution of the world standard population, stratified by sex and residential area (rural/urban).

Results ASMRs of stomach cancer were 15 per 100000 in men and 8 per 100000 in women. The highest and lowest mortality rates were observed in Kordestan (ASMR=29.1 per 100000 ) and Hormozgan $(A S M R=5.0$ per 100000$)$ provinces in the north-western and southern Iran, respectively. The mortality rates were approximately twice as high in men and rural residents as women and urban residents respectively.

Conclusions The incidence of stomach cancer in Iran is a high with evidence of regional variation. The substantial variation in stomach cancer mortality rates between northern and southern Iran warrants further investigation. The results of this study can be used for resource allocation and to inform the designing of appropriate gastric cancer control programs in Iran.

\section{P2-343 EFFECTS ANALYSIS OF THE PREVENTIVE MEASURES ON NEURAL TUBE DEFECTS IN CHINA}

doi:10.1136/jech.2011.142976k.75

Z Zhao, ${ }^{*}$ G Rui, Z Wang, L Gao, 0 Lu, X Sun. Department of Epidemiology, School of Public Health, Shandong University, Jinan, Shandong, China

Introduction China is among the high incidence countries for neural tube defects (NTDs) and great efforts have been made towards their prevention. This study is to understand the effects and influencing factors on prevention measures.
Methods A matched case-control study and prevalence studies were employed. 459 women who gestated babies/foetuses with NTDs and their controls were selected and investigated in 24 districts of two provinces in China. Logistic regression models and interaction analysis was used for data analysis.

Results Folic acid supplementation, planned pregnancy, preconception examination and health education were associated with reduced NTDs (ORs 0.52, 0.27, 0.48 and 0.36 respectively). The folic acid supplementation rate was $5.0 \%$ of cases and $17.2 \%$ of controls. Folic acid supplementation showed synergistic interaction effects with the other primary prevention measures and prevention rates were $93 \%, 89 \%$ and $90 \%$ respectively. $85.9 \%$ of the NTDs were diagnosed by ultrasound screening on average at 24.0 weeks gestation. The detection rates by ultrasonography before 16, 16-20, 20-24, 24-28 and after 28 weeks were $14.1 \%$, $49.4 \%, 46.3 \%, 49.2 \%$ and $52.1 \%$ respectively $(\mathrm{p}<0.05)$. The detection rates were $46.4 \%, 52.0 \%$ and $28.1 \%$ in hospitals, maternal and child care service centres and family planning centres respectively $(\mathrm{p}<0.05)$.

Conclusion Folic acid supplementation rate was low in the study subjects. Its use is correlated with planned pregnancy, preconception examination and health education. The efficiency of ultrasonography for NTDs screening could be improved in medical reproduction health institutions.

\section{P2-344 FRAILTY AND MULTIMORBIDITY IN THE ELDERLY: RESULTS FROM THE KORA-AGE AUGSBURG SURVEY}

doi:10.1136/jech.2011.142976k.76

${ }^{1} \mathrm{~A}-\mathrm{K}$ Zimmermann, ${ }^{*} \mathrm{~B}$ Thorand, ${ }^{1,2} \mathrm{C}$ Meisinger, ${ }^{1,2} \mathrm{M}$ Heier, ${ }^{1} \mathrm{~A}$ Peters, ${ }^{1} \mathrm{~A}$ Döring. ${ }^{1}$ Helmholtz Zentrum München, German Research Centre for Environmental Health, Institute of Epidemiology II, Neuherberg, Germany; ${ }^{2}$ Central Hospital of Augsburg, MONICA KORA Myocardial Infarction Registry, Augsburg, Germany

Background This study investigated the prevalence of frailty as well as its association with multimorbidity among older adults living in the South of Germany.

Methods 1079 participants aged 65 years and older were interviewed and took part in a physical examination in the KORAAge survey. Frailty was defined by weight loss, exhaustion, low physical activity, slow gait speed, and reduced grip strength. Participants were classified as non-frail, pre-frail, and frail if they met 0,1 or 2 , and 3 or more criteria, respectively. Multimorbidity was defined in three groups, group 1 with no disease, group 2 with one or two diseases, and group 3 with more than three out of nine disease groups. Multivariable logistic regression analyses adjusted for malnutrition, alcohol intake, education and body mass index were performed.

Results The age standardised prevalence of frailty and pre-frailty combined was $35.6 \%$ (95\% CI 31.1 to 40.0 ) for male and 38.5\% (95\% CI 34.1 to 42.8) for female participants. Prevalence of frailty increased with age from $24.1 \%$ in the $65-69$ year olds to $78.0 \%$ in the over 85 year olds for men ( $20.7 \%$ to $79.3 \%$ in women). The risk for being frail increased with higher multimorbidity scores (OR 1.9, $95 \%$ CI 1.4 to 2.6 for group 2 and OR $3.3,95 \%$ CI 1.8 to 6.0 for group 3 each compared to group 1) after adjustment for covariables. $27.8 \%$ of pre-frail and frail persons had no disease.

Conclusion These data indicate that frailty is strongly associated with multimorbidity. Examinations of the course of frailty and its determinants are underway. 